\title{
Evoked cardiac waveform components during aversion and detection procedures
}

\author{
DAVID FRIEDMAN \\ New York State Psychiatric Institute, New York, New York 10032 \\ BERNARD TURSKY \\ State University of New York at Stony Brook, Stony Brook, New York 11790 \\ and \\ L. ERLENMEYER-KIMLING \\ New York State Psychiatric Institute, New York, New York 10032
}

\begin{abstract}
This study assessed the nature of evoked cardiac components elicited during detection and aversion procedures. In the aversion procedure (AVR), tone intensity increased during the trial sequence, and the subject was instructed that he/she could stop the sequence if the intensity became uncomfortable. In the detection procedure (DET), the subject had to indicate during which of two observation intervals he/she had heard the tone. In this task, intensity decreased over the course of the experiment. A third condition (NOT), during which stimuli were not delivered, served as a baseline. Heart rate was sampled at three time periods (early, middle, and late) during the three conditions. The baseline condition produced no consistent cardiac response patterns. While the initial deceleratory and subsequent acceleratory components increased from early to late periods for DET, the AVR waveforms went from a small, initial deceleration to a wholly acceleratory response from early to late periods. These data provide further evidence that the initial, deceleratory portion of the cardiac waveform reflects "information intake," while the subsequent, acceleratory component reflects information processing.
\end{abstract}

The cardiac evoked response to simple stimuli consists of an initial, brief deceleratory component, followed by a longer lasting acceleration (e.g., Coles \& Duncan-Johnson, 1975; Graham \& Clifton, 1966; Lacey \& Lacey, 1970). Graham and Clifton (1966) suggested that the initial deceleration was indicative of orienting and signaled the intake of sensory information, whereas the acceleratory component was defensive in nature. This latter proposition has been supported by the work of Bull and Lang (1972), Graham and Slaby (1973), and Uno and Grings (1965), who showed increased accelerative activity with increases in stimulus intensity.

Graham and Clifton's (1966) argument was based on the cardiac response to nonsignal stimuli. The results from warned reaction time and detection tasks complicate this picture of the two-component model

The authors would like to thank: James Wilson, Jr., for aid with data collection and scoring, and Georgia Lenoil for her help with data scoring; James Hollenberg, for computer programs used in this study; David Lykken, who suggested we use the detection procedure and provided us with the details of that task; John Andreassi and Fred Rist, whose comments on an earlier version of this manuscript were most helpful; and Donald Ross, who provided us with invaluable statistical advice. This research was supported by USPHS Grant MH 19560 and by the Department of Mental Hygiene of the State of New York. of the cardiac waveform (cf. Coles \& Duncan-Johnson, 1975; Lacey \& Lacey, 1970). In tasks in which processing complexity and detection requirements are manipulated, the magnitude of the deceleratory component may reflect the enhancement of sensory sensitivity as this component increases with decreases in stimulus detectability (e.g., Coles, 1972; Coles \& Duncan-Johnson, 1975, 1977) and task difficulty (Kahneman, Tursky, Shapiro, \& Crider, 1969; Tursky, Schwartz, \& Crider, 1970), while the acceleratory component may reflect the occurrence of information processing as its magnitude increases with increments in processing complexity (e.g., Coles \& DuncanJohnson, 1975, 1977; Walter \& Porges, 1976) and stimulus uncertainty (Higgins, 1971).

The orienting vs. task-dependent explanations of these cardiac waveform components are not mutually exclusive. Rather, the amount of variance accounted for by either will depend on the nature of the experimental situation. We employed two procedures in which the nature of orienting and processing requirements was quite different. One was an aversion threshold procedure in which the intensity of a train of tones increased up to $105 \mathrm{~dB}$ SPL, and the subject was instructed that he/she could stop the stimuli if the intensity became uncomfortable. The other was a forced-choice auditory detection threshold procedure 
in which the tone decreased in intensity, and the subject was instructed to report during which of two observation intervals he/she had heard the tone. During the aversion procedure, the cardiac waveform should reflect orienting-defensive reactions, while during detection, component variation should be more dependent on signal detectability and processing requirements. In view of the differences in the intensity variable between tasks (aversion = increases; detection $=$ decreases) and the importance of trial sequence on habituation of the orienting response, the cardiac waveform was measured at three time periods during each procedure from early to late in the trial sequence, since one might expect differential task-related changes in these components as a function of time.

The two procedures formed part of a battery of tests designed to assess psychophysiological functioning in children of nonpsychiatrically disturbed parents and children of schizophrenic parents (high-risk children). Procedures similar to these had been shown to behaviorally differentiate adult schizophrenics from normal controls (Levine \& Whitney, 1970), while recent evidence has pointed to a reduction in deceleratory responding during these kinds of procedures in adult schizophrenics as compared with normal controls (Waddington, Maccullough, Schalker, \& Sambrooks, 1978; Zahn, Note 1). Our purpose in the present study was to determine the nature of the cardiac response elicited during these procedures in our sample of normal control children, and to develop measures of this activity that might later distinguish high-risk from normal control children.

\section{METHODS}

\section{Subjects}

Subjects were eight male and seven female adolescents ranging in age from 12 to 18 (mean $=13.8$ years) who formed part of a normal control group in a longitudinal high-risk project (ErlenmeyerKimling, Note 2). The children were given a 2.5-h psychophysiology battery, which included the two threshold procedures described below.

\section{Apparatus and Recording Procedure}

The electrocardiogram was recorded from right-leg and leftarm placements via Beckman biopotential skin electrodes with a Beckman Type 9806A preamplifier and Type 482M8 power amplifier on a Beckman Type RM dynograph. Stimulus presentation and data acquisition were under the control of a PDP $11 / 10$ computer, which digitized the EKG at 5-msec intervals and recorded it on digital tape for off-line analyses.

\section{Stimuli and Task Procedures}

Auditory stimuli were delivered binaurally, with rise and fall times of $2.5 \mathrm{msec}$, over TDH-39 300- $\Omega$ headphones from a Heathkit Model IG-18 generator through a General Radio Model 1287B electronic switch. Ambient noise in the experimental room was $64 \mathrm{~dB}$ SPL as measured by a Realistic sound-level meter.

Figure 1 depicts the events of one trial for AVR and DET and shows a small portion of the beginning of the next trial (marked 2 in the figure). The large vertical arrows at the beginning of each time line mark the start of a trial sequence and also mark the onset of heart period averaging. Both trial of fset and the end of averaging are indicated by downward arrows. For both AVR and DET, a trial lasted $10 \mathrm{sec}$.

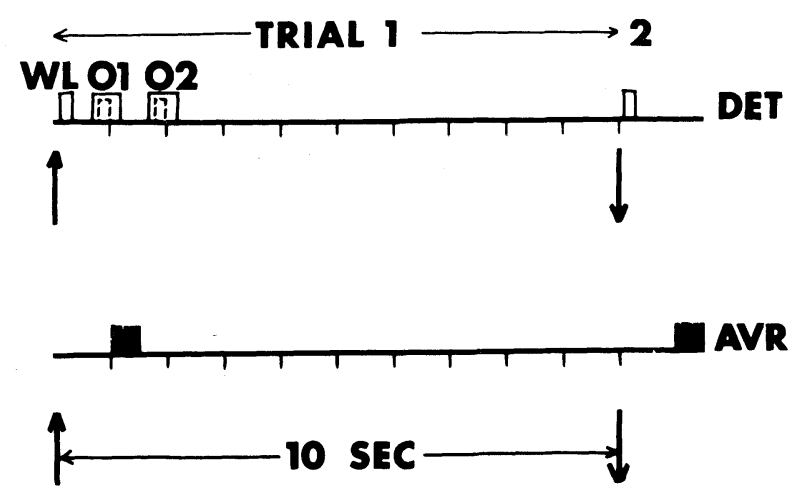

Figure 1. Trial events for the AVR and DET procedures. Arrows at the beginning of each time line mark the start of a trial and the onset of heart period averaging. Downward arrows mark the end of a trial and the end of the averaging period. The 10-sec period corresponds to Trial 1 , and the 2 refers to the onset of the next trial. Bars in Trial 2 mark the onset of the warning light for DET and the tone series for AVR. WL= warning light; 01 and 02 = observation intervals 1 and 2 . Hash marks indicate every second.

For AVR, a trial began with a 1 -sec prestimulus period, after which the subject heard a series of five $80-\mathrm{msec} 1,000-\mathrm{Hz}$ tones, with 20 -msec dead times between each tone (indicated by the five vertical bars on the AVR time line). The vertical bars in Trial 2 of AVR mark the onset of the next series of tone pulses. The time between two successive tone bursts was $10 \mathrm{sec}$.

The subject was instructed that the tones would increase in intensity and that he/she could stop the stimuli by pressing a response button if the tones became uncomfortably loud. The subjects were not required to respond except when they did not want to continue the task. The tone sequence started at $57 \mathrm{~dB}$ SPL and increased by $3 \mathrm{~dB}$ on each trial to a maximum of $105 \mathrm{~dB}$. The subjects included for study were chosen on the basis that they had completed the 17-trial sequence without interrupting the tones. Two subjects were eliminated because they did not complete the tone series.

A trial in the DET procedure began with a $100-\mathrm{msec}$ prestimulus period. This was followed by a $300-\mathrm{msec}$ warning light (WL in Figure 1), which served to alert the subject that a tone was going to be presented. The $1,000-\mathrm{Hz}$ tone was $200 \mathrm{msec}$ in duration (delivered as described above and indicated by the dashed lines in Figure 1) and was programmed to occur randomly during one of the two 500 -msec observation intervals ( 01 and 02 in Figure 1). The tone occurred $100 \mathrm{msec}$ after the onset of the observation interval. The time between observation intervals was $500 \mathrm{msec}$, with the first one occurring $300 \mathrm{msec}$ after the end of the warning light. The time between successive warning lights was $10 \mathrm{sec}$, and the vertical bar in Trial 2 of the DET time line marks the onset of the next warning light.

The two observation periods and the warning light were signaled by light-emitting diodes (LEDs), which were mounted on a panel in front of the subject. The two observation LEDs had associated response keys mounted below them, and the subject was instructed to pay close attention and to press the key (following the offset of the second observation interval) that corresponded to the interval during which he/she had heard the tone.

The subjects were instructed to guess if they were not sure. Tone intensity began at $45 \mathrm{~dB}$ SPL and was decreased $1 \mathrm{~dB}$ for every correct response and increased $4 \mathrm{~dB}$ for every incorrect response (i.e., when the subject pressed a key that corresponded to the observation interval during which the tone had not occurred). The procedure ended after the subject had made five incorrect responses. Threshold was taken as the mean of the decibel levels corresponding to the five errors. All the subjects received a no-stimulus, baseline run (NOT), which corresponded 
to 17 10-sec "trials," followed by the AVR and then the DET procedures.

\section{Data Analyses}

The data were played back from tape and written out for 10 sec periods (one trial) onto a Hewlett-Packard X-Y recorder. These plots were scored for successive $R-R$ intervals starting from the onset of the trial. For DET only, those trials in which a correct response had occurred were included for analysis. In order to determine the effect of time, each of the three procedures (NOT, AVR, DET) was divided into early, middle, and late periods, and three trials of $R-R$ intervals were averaged for each period. For NOT and AVR, the selected trials were 1-3, 7-9, and 15-17 for all subjects, but for DET, the selection of middle and late trials depended upon how long it took the subject to make five errors. The early period was always represented by Trials 1-3. For these subjects, the range of trials for DET was 27-65, with a mean of 38. The intensity of the tone decreased over time and was at a mean of $44 \mathrm{~dB}$ for the early trials, $34 \mathrm{~dB}$ for the middle trials, and $24 \mathrm{~dB}$ for the late trials. For AVR, tone intensity was at a mean of $60 \mathrm{~dB}$ for early, $78 \mathrm{~dB}$ for middle, and $102 \mathrm{~dB}$ for late trials. For the majority of subjects, the first full beat for AVR spanned the pre- and posttone onset periods. For DET, the first full beat for the majority of subjects spanned the postwarning light and first observation periods.

There were nine averaged evoked cardiac waveforms for each subject, corresponding to the three time periods within each of the three procedures.

All data to be reported are in terms of heart period and cardiac time. Recent evidence has shown that heart period meets more of the assumptions of the most frequently used statistical tests (Jennings, Stringfellow, \& Graham, 1974; Khatchaturian, Kerr, Kruger, \& Schachter, 1972), and the combination of heart period and cardiac time meets the assumption of yielding unbiased means (Graham, 1978).

To remove level differences that were obvious at Beat 1 , this beat was subtracted from all subsequent beats for each subject.

Repeated measures ANOVAs (BMDP2V, Dixon, 1975) were used to assess the effects of task and time period on the cardiac waveform. The form of the response over beats and differences in this form as a function of task and time period were assessed by orthogonal polynomials using unpooled error terms. These tests were not, therefore, subject to the bias due to unequal covariance among repeated measures (Graham, 1971). Since tests over all beats will reflect this bias, the degrees of freedom for these tests were reduced, yielding a much more conservative test (Winer, 1971), according to the method outlined by Jennings and Wood (1976).

\section{RESULTS}

\section{Cardiac Waveforms}

The grand mean difference curves are displayed in Figure 2. Subjects varied in the number of beats produced during each 10 -sec period, from a low of 7 to a high of 12. Therefore, we looked at seven beats across all subjects. The cardiac responses for NOT do not display any consistent trends across time periods. Both AVR and DET elicited initial deceleratory and subsequent acceleratory components whose amplitudes differed as a function of time period. For AVR, the amplitude of the acceleratory component does not appear to change with time, whereas the initial deceleration, which is of small amplitude for the first two time periods, is absent for the late period. For DET, there is no initial deceleration and a moderate acceleratory component for the
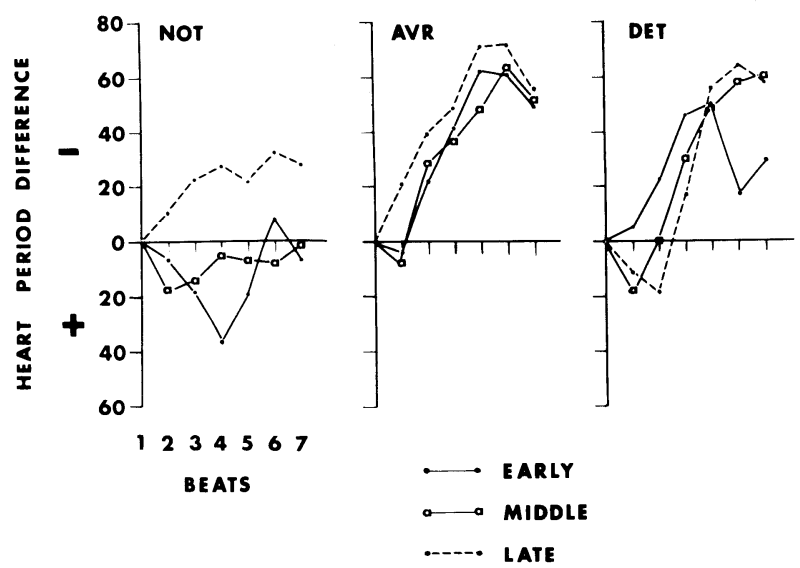

Figure 2. Grand mean difference cardiac waveforms across the 15 subjects, plotted for each combination of task and time period for seven beats.

early period, while the middle and late period responses show larger initial decelerations and larger subsequent accelerations.

\section{Trend Analyses}

To assess differences in response form as a function of task and time, trend analyses were performed on the seven beats, separately for each task and time period. The results of these analyses appear in Table 1 . With the exception of a significant quartic component at the early time period, NOT showed no significant response trends. In contrast, both AVR and DET showed highly significant trend components, which differed depending upon the time period. For AVR, the significant linear trends at all time periods reflected the large acceleratory components seen in these waveforms. The quadratic and cubic trends were sig-

Table 1

F Ratios and Significance of Seven-Beat Response Trends for Early (E), Middle (M), and Late (L) Time Periods

\begin{tabular}{lcccc}
\hline \multirow{2}{*}{ Trend } & $\begin{array}{c}\text { Time } \\
\text { Period }\end{array}$ & NOT & AVR & DET \\
\hline \multirow{4}{*}{ Linear } & $\mathrm{E}$ & .03 & $11.29 \dagger \dagger$ & 1.61 \\
& $\mathrm{M}$ & .64 & $10.86 \dagger$ & $25.91 \ddagger$ \\
& $\mathrm{L}$ & .14 & $16.52 \dagger \dagger$ & $16.02 \dagger \dagger$ \\
Quadratic & $\mathrm{E}$ & .10 & $4.85^{*}$ & $4.62^{*}$ \\
& $\mathrm{M}$ & .53 & 2.07 & .05 \\
& $\mathrm{~L}$ & .33 & 2.95 & .68 \\
Cubic & $\mathrm{E}$ & .61 & $4.88^{*}$ & .09 \\
& $\mathrm{M}$ & .44 & 2.50 & 3.78 \\
& $\mathrm{~L}$ & .75 & .57 & $7.50^{* *}$ \\
Quartic & $\mathrm{E}$ & $7.89 * *$ & .27 & 4.34 \\
& $\mathrm{M}$ & .32 & .20 & .88 \\
& $\mathrm{~L}$ & .97 & .34 & .19 \\
\hline
\end{tabular}

Note $-d f=1,14$.

${ }^{*} p<.05 . \quad{ }^{* *} p<.025 . \quad \dagger p<.01 . \quad+\dagger p<.005 . \quad \ddagger p<.001$. 
nificant only at the early period, reflecting the small initial and longer latency decelerations seen at this period. For AVR, the cubic component decreases with time, reflecting the change to a wholly acceleratory response pattern. The early DET waveform produced only a significant quadratic trend and marginally significant quartic trend, reflecting the smaller acceleratory and larger subsequent deceleratory components and the very late (Beat 7) acceleration, as compared with middle and late waveforms. Both middle and late DET waveforms showed large linear trends reflecting their large-amplitude acceleratory components. In contrast with AVR, the DET cubic trend increased with time, indicating a change to a more prominent initial deceleration.

\section{Task by Time by Beat Comparisons}

A comparison of the beat-by-beat cardiac response patterns across the three tasks and time periods was made using a 3 (NOT, AVR, DET) by 3 (early, middle, late) by 7 (beats) ANOVA. In addition to a beat effect $[F(6,84$ reduced to 1,14$)=17.23, p<.001]$, the Task by Beat interaction confirmed that the three evoked cardiac waveforms (collapsed across time periods) were statistically different $[F(12,168$ reduced to 1,14$)=4.32, p<.05^{1}$ ]. Tests for simple effects (Winer, 1971) of beats for each task showed that NOT did not produce significant variation with beats, while both AVR $[F(1,14)=18.94, p<.001]$ and DET $[F(1,14)=21.80, p<.001]$ did. Simple effects of task at each beat showed that, with the exception of Beat 2 , task was a significant source of variation [all Fs $(1,14>$ $4.60, p<.05$ or less]. Newman-Keuls procedures at each beat showed that these significant sources of variance were due to greater acceleration at Beats 4-7 for AVR and DET than for NOT, while AVR and DET did not differ. At Beat 3, AVR showed greater acceleration than both DET and NOT, which did not differ from each other.

Although the interaction of task, time period, and beats was not significant $[F(24,366$ reduced to 1,14$)$ $=1.33, \mathrm{p}>.05$ ], the previous trend analyses had shown that the cardiac waveform differed as a function of these variables. Because of these differences in trend and our interest in waveform change over time, tests for simple effects of this interaction were performed. At each time period, both AVR and DET showed significant variation with beats [all Fs $(1,14)$ $>10, p<.01$ or less], while NOT showed significant variation with beats at the early period only $[F(1,14)$ $=8.73, \mathrm{p}<.025]$.

Tests for simple effects of task at each beat showed significant variation due to task at all time periods, with the exception of Beat 2 at early and middle periods [all $\mathrm{Fs}(1,14)>4.60$, $\mathrm{p}<.05$ or less]. NewmanKeuls procedures showed that at early and middle periods, AVR and DET showed significantly greater acceleratory patterns than NOT, but did not differ from each other. At the late period, for Beats 2 and 3, AVR and DET differed significantly due to the fact that DET showed deceleration and AVR showed acceleration, while at Beats 4 through 7, both AVR and DET showed equivalent responses.

NOT showed significant changes with time [all Fs $(1,14)>5, p<.05$ or less], due primarily to a change from deceleration at early periods to acceleration at late periods (Newman-Keuls procedures showed that late means differed significantly from early means, while middle and early and middle and late did not). AVR showed no significant variation as a function of time. For DET, Beats 3, 6, and 7 showed significant variation with time [all $\operatorname{Fs}(1,14)>5, \mathrm{p}<$ .05 or less], and Newman-Keuls procedures showed that this was due to a change from acceleration to deceleration from early to late periods for Beat 3 and a change to greater acceleration from early to late periods for Beats 6 and 7.

\section{DISCUSSION}

The present results indicate that the initial deceleratory component of the cardiac waveform is elicited when the experimental situation requires subjects to pay close attention to task-relevant stimuli. This component grew in amplitude in the DET curves as the tone approached threshold level, but decreased in the AVR waveforms as the tone approached maximum intensity. The subsequent acceleratory component increased in amplitude from early to late periods for DET, but did not change with time for the AVR waveforms. Thus, the same components were elicited by both procedures, but were dissociable on the basis of their relationship with time and task requirements.

The cardiac response to simple stimuli has been extensively studied, and the results have indicated that morphology and component amplitude depend upon both the quality and intensity of the eliciting stimulus (e.g., Graham \& Slaby, 1973; Turpin \& Siddle, 1978). All of the investigators employing tonal stimuli (even at high intensities), with no task imposed on the subject, have reported finding response curves with three components (initial deceleration, subsequent acceleration, and a late deceleration), while those using white noise have shown that these stimuli produce only two components (acceleration and deceleration), even at lower intensities. The morphologies of the responses in the present experiment conform to those reported by others over the range of intensities used here.

The introduction of a task does not change the morphology of the cardiac response; rather, it changes the amplitude of the primary components (e.g., Coles \& Duncan-Johnson, 1977; Walter \& Porges, 1976). Analysis of the evoked responses collapsed across 
time periods showed significant effects of task, with NOT showing no variation with beats, AVR producing only acceleration and a late, low-amplitude deceleration, and DET showing an initial, brief deceleration followed by a large-amplitude acceleration.

The most consistent differences between the AVR and DET waveforms were seen when trial sequence was examined as an interactive variable with task and beats. AVR and DET showed equivalent acceleratory responses at early and middle periods but differed at the late period. At this time period, DET showed an initial deceleration, while AVR showed acceleration. DET produced a change to a larger amplitude acceleratory component from early to late periods and a significant increase in initial deceleration, while AVR showed no such variation with time. As the subject approaches threshold in DET, discrimination of the tone from noise becomes more difficult, requiring greater attentiveness, and this results in an increase in initial deceleration from early to late periods. Coles and Duncan-Johnson $(1975,1977)$ and Schell and Catania (1975) have shown that deceleration accompanies activity related to detection and varies with the degree of difficulty of the task. It is also likely that uncertainty as to the presence of the tone creates more of an information load as tone intensity decreases, which is reflected in the increase in acceleratory responding. Support for this assertion comes from studies by Connor and Lang (1969), Higgins (1971), and Walter and Porges (1976), who have shown that the amplitude of the acceleratory component following stimulus onset varies with the complexity of information processing.

The possibility must be raised here that motor response requirements that differed between the two procedures may have accounted for the cardiac waveform differences seen between DET and AVR. This seems unlikely, as the same motor response was required on each DET trial (following the second observation interval), and it would, therefore, be difficult to account for the changes in the DET components on this basis.

The cardiac waveform elicited under AVR showed a small, initial deceleration at the early period, while for the late period, the response consisted entirely of a large-amplitude acceleration. This might be expected if the response changes from orienting to defensive as intensity increases. Graham and Slaby (1973) found no change with repetition in the acceleratory component to an $85-\mathrm{dB}$ tone, but found a marked amplitude diminution of the initial acceleratory component to an $85-\mathrm{dB}$ white noise stimulus. Turpin and Siddle (1978) reported finding a larger acceleratory component to a $110-\mathrm{dB}$ white noise than to a $60-\mathrm{dB}$ white noise. In the present study, the acceleratory component elicited by AVR did not differ with time, and this is most likely due to the fact that intensity influenced the amplitude of this component more than did habituation. Our data, in conjunction with those of Graham and Slaby (1973) and Turpin and Siddle (1978), also suggest that a tone at intense-enough levels may elicit an accelerative response normally seen only with white noise stimuli.

A possible confounding in the present study arises from the fact that task order was not counterbalanced (DET always followed AVR, which always followed NOT), and DET and AVR were not equated for number of trials. On these bases, it would be expected that both the deceleratory and acceleratory components elicited during DET would diminish with time (i.e., would be most affected by habituation). However, both components grew in amplitude with time, the opposite of what would be predicted had habituation greatly influenced component amplitude. For AVR, it is possible that habituation had an effect. However, subjective reports reflecting the uncomfortable nature of the tones at the higher intensities are incompatible with this view and make it likely that the changes seen in AVR as a function of time reflect a shift from orienting to defensive responding.

The results provide support for the data of Lacey and Lacey (1970) in showing that initial deceleration was exhibited primarily in the DET, and not the AVR, curves, a task in which the "noting and detecting", of environmental events were crucial. Both tasks produced large-amplitude acceleratory components-but only those for DET were larger for late, than for early, periods-at a time when information load was greatest.

These data provide further evidence that the components of the cardiac evoked response reflect distinct psychological processes whose elicitation depends upon stimulus characteristics and task requirements.

\section{REFERENCE NOTES}

1. Zahn, T. P. Heart rate, hyperactivity and schizophrenia. Paper presented at the Nineteenth Annual Meeting of the Society for Psychophysiological Research, Cincinnati, Ohio, October 1979.

2. Erlenmeyer-Kimling, L. A prospective study of children of schizophrenic parents. USPHS Grant MH-19560, 1970.

\section{REFERENCES}

But., K., \& Lana, P. J. Intensity judgments and physiological response amplitude. Psychophysiology, 1972, 9, 428-436.

Coles, M. G. H. Cardiac and respiratory activity during visual search. Journal of Experimental Psychologv, 1972, 96, 371-379.

Coles, M. G. H., \& Duncan-Johnson, C. Cardiac activity and information processing: The effects of significance, and detection and response requirements. Journal of Experimental Psvchologv: Human Perception and Performance, 1975, 1. $418-428$

Comlis. M. G. H., \& Duncan-Jomnson, C. Attention and cardiac 
activity: Heart rate responses during a variable foreperiod disjunctive reaction time task. Biological Psychologv, 1977, 5. $151-158$

Connor, W. H.. \& Lanci, P. J. Cortical slow-wave and cardiac rate responses in stimulus orientation and reaction time conditions. Journal of Experimental Psychology, 1969, 82, 310-320.

Dixon. W. J. BMDP computer programs. Los Angeles: University of California Press, 1975.

Grinam, F. K. Analysis of heart rate response curves: A comment on pooled interaction error terms. Psychophusiology, $1971,7,485-489$.

Granam, F. K. Constraints on measuring heart rate and period sequentially through real and cardiac time. Psychophysiolog.v, $1978,15,492-495$.

Granam, F. K., \& Cilfton, R. K. Heart rate change as a component of the orienting response. Psychological Bulletin, 1966, 65, 305-320.

Graham, F. K., \& Siaby, D. A. Differential heart rate change to equally intense white noise and tone. Psychophysiology, $1973,10,347-362$.

Hugians, J. D. Set and uncertainty as factors influencing anticipatory cardiovascular responding in humans. Journal of Comparative and Physiological Psychology, 1971, 74, 272-283.

Jenningis, J. R., Stringfellow, J. C., \& Graham, M. A comparison of the statistical distributions of beat-by-beat heart rate and heart period. Psychophysiology, 1974, 11, 207-210.

Jennings, J. R., \& Wood, C. C. The $\varepsilon$-adjustment procedure for repeated measures analyses of variance. Psychophysiology, 1976, 13, 277-278.

Kahneman, D., Tursky, B., Shapiro, D., \& Crider, A. Pupillary, heart rate and skin resistance changes during a mental task. Journal of Experimental Psychology, 1969, 79, 164-167.

Khachaturian, Z. S., Kerr, J., Kruger, R., \& Schachter, J. A methodological note: Comparison between period and rate data in studies of cardiac function. Psychophysiology, 1972, 9, 539-545.

LaCEy, J. I., \& Lacey, B. C. Some autonomic-central nervous system interrelationships. In P. Black (Ed.), Physiological correlates of emotion. New York: Academic Press, 1970.
Levinf, F. M., \& Whitnty, N. Absolute auditory threshold and threshold of unpleasantness of chronic schizophrenic patients and normal controls. Journal of A hnormal Psvchology. 1970, 75, 74-76.

Schell, A. M., \& Catania, J. The relationship hetween cardiac activity and sensory acuity. Psvchophvsiologv, 1975, 12, 147-151.

Turpin, G., \& Sindi., D. A. T. Cardiac and forearm plethysmographic responses to high intensity auditory stimulation. Biological Psychology, 1978, 6, 267-281.

Tursky, B., Schwartz, G. E., \& Crider, A. Differential patterns of heart rate and skin resistance during a digit transformation task. Journal of Experimental Psychology, 1970, 83, 451-457.

Uno, T., \& Grings, W. W. Autonomic components of orienting behavior. Psychophysiology, 1965, 1, 311-321.

Waddington, J. L., Macculloch, M. J., Schalker, M. C., \& SАмвгоокs, J. E. Absence of cardiac deceleration in a signalled escape paradigm: A psychophysiological deficit in chronic schizophrenia. Psychological Medicine, 1978, 8, 157-162.

Walter, G. F., \& Porges, S. W. Heart rate and respiratory responses as a function of task difficulty: The use of discriminant analysis in the selection of psychologically sensitive physiological responses. Psychophysiology, 1976, 13, 563-571.

Winfr, B. J. Statistical principles in experimental design. New York: McGraw-Hill, 1971.

\section{NOTE}

1. Reducing the degrees of freedom results in an extremely conservative test (Jennings \& Wood, 1976; Winer, 1971). Since the Task by Beat F-value was highly significant with full degrees of freedom and fell just short of significance $(4.60$ is necessary with $1,14 \mathrm{df}$ for $\mathrm{p}<.05$ ) with the conservative test, we are considering the interaction significant at the .05 level.

(Received for publication January 14, 1980; revision accepted April 12, 1980.) 